\title{
Status of avifauna at Taranga Hill-forest, Gujarat, India
}

\author{
C.D. Patel ${ }^{1} \&$ M.I. Patel ${ }^{2}$ \\ ${ }^{1}$ Department of Biology, ${ }^{2}$ Principal, \\ M.N. College, Visnagar, Gujarat 384315, India. \\ Email: ${ }^{1}$ chirag.naja@ gmail.com, ${ }^{2}$ prinmncvis@ gmail.com
}

Date of publication (online): 26 February 2010 Date of publication (print): 26 February 2010 ISSN $0974-7907$ (online) | $0974-7893$ (print)

Editor: M. Zornitza Aguilar

Manuscript details:

Ms \# 02278

Received 31 July 2009

Final received 09 January 2010

Finally accepted 11 February 2010

Citation: Patel, C.D. \& M.I. Patel (2010). Status of avifauna at Taranga Hill-forest, Gujarat, India. Journal of Threatened Taxa 2(2): 695-699.

Copyright: (C C.D. Patel \& M.I. Patel 2010. Creative Commons Attribution 3.0 Unported License. JoTT allows unrestricted use of this article in any medium for non-profit purposes, reproduction and distribution by providing adequate credit to the authors and the source of publication.

Author Details: C.D. PATEL, a research scholar is a life member of Vihang Research Foundation, Ahmedabad \& Bird Conservation Society, Gujarat. $\mathrm{He}$ is pursuing research in ornithology with special emphasis on terrestrial birds under the supervision of M.I. Patel.

M.I. PATEL's field of specialization is in population ornithology. At present, he is a member of the State level Expert Appraisal Committee, Gujarat. $\mathrm{He}$ is a life member of BNHS, ISCA, Kolkata \& WWF, Mumbai.

Author Contribution: CDP conducted fieldwork for two years, identified birds and conducted population counts. He has contributed towards writing and analysis of field data. MIP formulated and conceptualized the project, research design and plan of work.

Acknowledgements: We are thankful to Devarshi Bhavasar who helped us regularly throughout our field work without any self-interest. C.D.P. is thankful to the Commissioner of Higher Education, Gandhinagar for financial support.
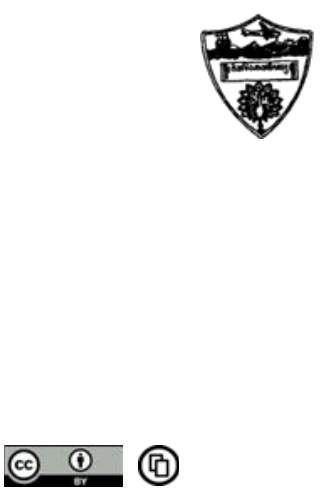

OPEN ACGESS | FREE DOWNLOAD
Abstract: Taranga is one of the famous pilgrim places of northern Gujarat. It is located $\left(24^{\circ} 00^{\prime} \mathrm{N}\right.$ $\left.\& 72^{\circ} 46^{\prime} \mathrm{E}\right)$ at starting point of Aravalli ranges. Climate of this area is semi-arid with irregular rainfall. Variable width line transect method was adapted to study the avifaunal diversity. Taranga Hillforest has atleast 90 species of birds belonging to 11 orders, 33 families and 68 genera. Passeriformes being the largest family. All common residents appear to be adapted to the prevailing conditions. Red-vented Bulbul and Rock Pigeon were most abundant while Asian Paradiseflycatcher, Crested Bunting and European Roller were rare. White-naped Tit a globally threatened and endemic resident has been found as local migrant, scarce in number, common in occurrence and breeder in the tropical thorn-scrub habitat of THf. Plum-headed Parakeet may be a breeding possible species. In comparison to other places, the avian diversity is observed poor, because Aravallis are not on the migratory route or landing site of migratory birds. In addition, anthropogenic factors, presence of predators and loss of vegetation may be having a telling effect.

Keywords: Aravalli ranges, avifauna, breeding possible, breeding probable, migrant, omnivores.

\section{INTRODUCTION}

Avian community studies are effective tools for monitoring a forest ecosystem. Birds are widely recognized as good bio-indicators of the quality of the ecosystems (Gill 1994) and health of the environment. They are responsive to change; their diversity and abundance can reflect ecological trends in other biodiversity (Furness \& Greenwood 1993). Because of their highly specific habitat requirements, birds become increasingly intolerant of even slight ecosystem disturbance (Schwartz \& Schwartz 1951).

Work on forest bird community has been done in other parts of the country time to time. Ramakrishnan (1983) examined several parameters of the bird communities in the forests of northern Kerala. Diversity and community structure of birds were also studied by Johnsingh et al. (1987), Johnsingh \& Joshua (1994), Katti (1989), Daniels (1989, 1996 \& 1997), Gokula \& Vijayan (1996) and Sundaramoorthy (1991). In similar lines an attempt has been made to study and assess the present status of birds in the Taranga Hill-forest.

\section{Study Area}

Taranga is one of the famous pilgrim places of northern Gujarat. The Taranga Hillforest (THf) is located at starting point of Aravalli ranges and situated at $24^{\circ} 00^{\prime} \mathrm{N}$ \& $72^{\circ} 46^{\prime} \mathrm{E}(365.76 \mathrm{~m})$ in the northern Gujarat region, India. The THf is one of the unclassified reserve forests (under section-IV) with total area of $18.12 \mathrm{~km}^{2}$. According to Champion \& Seth (1968), the THf falls in to forest type 5/E2 (Boswellia type of forest) of northern Gujarat.

Climate of the area is semi-arid with irregular rainfall. It is strongly periodical and seasonal. There are three main seasons: Winter (November-February), Summer (March-June) and Monsoon (July-October). Winter is the period of cold weather. Worm dry weather remains during summer. The hottest month of the year is May. Westerly to south-westerly winds prevails during monsoon. Variable climate has experienced through out the year, which mainly affects on vegetation and arthropod abundance.

Taranga Hill-forest experiences a prolonged dry season. Average temperature remains $19.80^{\circ} \mathrm{C}$ to $30.73^{\circ} \mathrm{C}$. The dry season is characteristic by low and erratic rain received primarily during the monsoon (months of July to September). It gets most of its rain from the south-west monsoon, which usually sets in by the middle of June or the beginning of July and continues until September and at times until the beginning of October. Heavy rain occurs during July and August but usually remains light during June and September. Average annual rainfall remains $663.60 \mathrm{~mm}$ with about 40 rainy days. 
Table 1. Systematic list and status of avifauna at Taranga Hill-forest

\begin{tabular}{|c|c|c|c|c|c|c|}
\hline \multirow[t]{2}{*}{ Order, Family \& Common name } & \multirow[t]{2}{*}{ Scientific name } & \multirow[b]{2}{*}{ Resident } & \multirow[b]{2}{*}{ Abundant } & \multicolumn{2}{|l|}{ Status } & \multirow[b]{2}{*}{$\begin{array}{l}\text { Primarily } \\
\text { feeding habit }\end{array}$} \\
\hline & & & & Occurrence & Breeding & \\
\hline \multicolumn{7}{|l|}{$\begin{array}{l}\text { Galliformes } \\
\text { Phasianide }\end{array}$} \\
\hline 1. Grey Francolin & Francolinus pondicerianus & $\mathrm{R}$ & $\mathrm{F}$ & $\mathrm{FC}$ & B & GR \\
\hline 2. Indian Peafowl & Pavo cristatus & $\mathrm{R}$ & Lf & $\mathrm{FC}$ & $\mathrm{B}$ & $\mathrm{OM}$ \\
\hline \multicolumn{7}{|l|}{$\begin{array}{l}\text { Piciformes } \\
\text { Picidae }\end{array}$} \\
\hline 3. Yellow-crowned Woodpecker & Dendrocopos mahrattensis & $\mathrm{R}$ & Sc & $\mathrm{FC}$ & $\mathrm{BP}$ & IN \\
\hline 4. Black-rumped Flameback & Dinopium benghalense & LM & $\mathrm{Sc}$ & $\mathrm{FC}$ & $?$ & IN \\
\hline \multicolumn{7}{|l|}{ Megalaimidae } \\
\hline 5. Coppersmith Barbet & Megalaima haemacephala & LM & Sc & $\mathrm{FC}$ & $\mathrm{BP}$ & FR \\
\hline \multicolumn{7}{|l|}{$\begin{array}{l}\text { Upupiformes } \\
\text { Upupidae }\end{array}$} \\
\hline 6. Common Hoopoe & Upupa epops & WM & Lf & $\mathrm{O}$ & NB & IN \\
\hline \multicolumn{7}{|l|}{$\begin{array}{l}\text { Coraciiformes } \\
\text { Coraciidae }\end{array}$} \\
\hline 7. Indian Roller & Coracias benghalensis & $\mathrm{R}$ & Lf & $\mathrm{FC}$ & B & IN \\
\hline 8. European Roller & Coracias garrulus & PM & Sc & r & NB & IN \\
\hline \multicolumn{7}{|l|}{ Dacelonidae } \\
\hline 9. White-throated Kingfisher & Halcyon smyrnensis & $\mathrm{R}$ & Sc & $\mathrm{FC}$ & $\mathrm{B}$ & $\mathrm{PI}$ \\
\hline \multicolumn{7}{|l|}{ Meropidae } \\
\hline 10. Green Bee-eater & Merops orientalis & $\mathrm{R}$ & $\mathrm{F}$ & $\mathrm{FC}$ & B & IN \\
\hline 11. Blue-cheeked Bee-eater & Merops persicus & WM & Lf & $\mathrm{O}$ & NB & IN \\
\hline \multicolumn{7}{|l|}{$\begin{array}{l}\text { Cuculiformes } \\
\text { Cuculidae }\end{array}$} \\
\hline 12. Asian Koel & Eudynamys scolopacea & $\mathrm{R}$ & Lf & $\mathrm{FC}$ & $\mathrm{B}$ & $\mathrm{FR}$ \\
\hline 13. Pied Cuckoo & Clamator jacobinus & MM & Lf & $\mathrm{O}$ & NB & IN \\
\hline \multicolumn{7}{|l|}{ Centropodidae } \\
\hline 14. Greater Coucal & Centropus sinensis & $\mathrm{R}$ & Lf & $\mathrm{FC}$ & $\mathrm{B}$ & $\mathrm{CR}$ \\
\hline \multicolumn{7}{|l|}{$\begin{array}{l}\text { Psittaciformes } \\
\text { Psittacidae }\end{array}$} \\
\hline 15. Plum-headed Parakeet & Psittacula cyanocephala & $\mathrm{R}$ & $\mathrm{F}$ & $\mathrm{FC}$ & PB & $\mathrm{FR}$ \\
\hline 16. Rose-ringed Parakeet & Psittacula krameri & $\mathrm{R}$ & La & $\mathrm{FC}$ & $\mathrm{B}$ & FR \\
\hline \multicolumn{7}{|l|}{$\begin{array}{l}\text { Apodiformes } \\
\text { Apodidae }\end{array}$} \\
\hline 17. House Swift & Apus affinis & $\mathrm{R}$ & La & $\mathrm{FC}$ & $\mathrm{B}$ & IN \\
\hline \multicolumn{7}{|l|}{$\begin{array}{l}\text { Strigiformes } \\
\text { Strigidae }\end{array}$} \\
\hline 18. Spotted Owlet & Athene brama & $\mathrm{R}$ & Lf & $\mathrm{FC}$ & $\mathrm{B}$ & $\mathrm{CR}$ \\
\hline \multicolumn{7}{|l|}{$\begin{array}{l}\text { Columbiformes } \\
\text { Columbidae }\end{array}$} \\
\hline 19. Rock Pigeon & Columba livia & $\mathrm{R}$ & A & $\mathrm{FC}$ & B & GR \\
\hline 20. Eurasian Collared Dove & Streptopelia decaocto & $\mathrm{R}$ & $\mathrm{F}$ & $\mathrm{FC}$ & $\mathrm{B}$ & GR \\
\hline 21. Laughing Dove & Streptopelia senegalensis & $\mathrm{R}$ & $\mathrm{F}$ & $\mathrm{FC}$ & B & GR \\
\hline 22. Yellow-footed Green Pigeon & Treron phoenicoptera & $\mathrm{R}$ & $\mathrm{F}$ & $\mathrm{FC}$ & NB & $\mathrm{FR}$ \\
\hline $\begin{array}{l}\text { Ciconiiformes } \\
\text { Burhinidae }\end{array}$ & & & & & & \\
\hline 23. Eurasian Thick-knee & Burhinus oedicnemus & LM & Sc & $\mathrm{FC}$ & $\mathrm{BP}$ & $\mathrm{CR}$ \\
\hline $\begin{array}{l}\text { Charadriidae } \\
\text { 24. Red-Wattled Lapwing }\end{array}$ & Vanellus indicus & $\mathrm{R}$ & Lf & $\mathrm{FC}$ & B & IN \\
\hline $\begin{array}{l}\text { Accipitridae } \\
\text { 25. Shikra }\end{array}$ & Accipiter badius & $\mathrm{R}$ & Lf & $\mathrm{FC}$ & $\mathrm{B}$ & $\mathrm{CR}$ \\
\hline 26. White-eyed Buzzard & Butastur teesa & LM & Sc & C & $\mathrm{BP}$ & $\mathrm{CR}$ \\
\hline 27. Black-Shouldered Kite & Elanus caeruleus & $\mathrm{R}$ & Lf & $\mathrm{FC}$ & B & $\mathrm{CR}$ \\
\hline 28. Black Kite & Milvus migrans & LM & Sc & C & $\mathrm{B}$ & OM \\
\hline 29. Oriental Honey-buzzard & Pernis ptilorhyncus & $\mathrm{R}$ & Lf & $\mathrm{FC}$ & $\mathrm{B}$ & $\operatorname{IN}(?)^{*}$ \\
\hline $\begin{array}{l}\text { Podicipedidae } \\
\text { 30. Little Grebe }\end{array}$ & Tachybaptus ruficollis & LM & Sc & 0 & $\mathrm{BP}$ & $\operatorname{IN}(?)^{\star}$ \\
\hline Ardeidae & & & & & & \\
\hline 31. Indian Pond Heron & Ardeola grayii & LM & Sc & $\mathrm{O}$ & $\mathrm{BP}$ & $\mathrm{PI}(?)^{\star}$ \\
\hline 32. Cattle Egret & Bubulcus ibis & $\mathrm{R}$ & Lf & $\mathrm{FC}$ & B & IN \\
\hline 33. Great Egret & Casmerodius albus & LM & Sc & 0 & $\mathrm{BP}$ & PI \\
\hline 34. Intermediate Egret & Mesophoyx intermedia & LM & $\mathrm{Sc}$ & $\mathrm{O}$ & $\mathrm{BP}$ & $\mathrm{PI}$ \\
\hline Threskiornithidae & & & & & & \\
\hline 35. Black Ibis & Pseudibis papillosa & LM & Sc & C & B & IN \\
\hline 36. Black-headed Ibis & Threskiornis melanocephalus & LM & Sc & 0 & B & $\operatorname{IN}(?)^{\star}$ \\
\hline
\end{tabular}




\begin{tabular}{|c|c|c|c|c|c|c|}
\hline \multirow[t]{2}{*}{ Order, Family \& Common name } & \multirow[t]{2}{*}{ Scientific name } & \multicolumn{5}{|c|}{ Status } \\
\hline & & Resident & Abundant & Occurrence & Breeding & $\begin{array}{l}\text { Primarily } \\
\text { feeding habit }\end{array}$ \\
\hline \multicolumn{7}{|l|}{$\begin{array}{l}\text { Passeriformes } \\
\text { Laniidae }\end{array}$} \\
\hline 37. Southern Grey Shrike & Lanius meridionalis & $\mathrm{R}$ & $\mathrm{F}$ & $\mathrm{FC}$ & B & IN $(?)^{*}$ \\
\hline 38. Long-tailed Shrike & Lanius schach & $\mathrm{R}$ & Lf & $\mathrm{FC}$ & $B$ & IN $(?)^{*}$ \\
\hline \multicolumn{7}{|l|}{ Corvidae } \\
\hline 39. Marshall's Lora & Aegithina nigrolutea & $\mathrm{R}$ & Lf & $\mathrm{FC}$ & $B$ & IN \\
\hline 40. Common Lora & Aegithina tiphia & $\mathrm{R}$ & Lf & $\mathrm{FC}$ & $?$ & IN \\
\hline 41. Large-billed Crow & Corvus macrorhynchos & $\mathrm{R}$ & Lf & $\mathrm{FC}$ & NB & $\mathrm{OM}$ \\
\hline 42. House Crow & Corvus splendens & $\mathrm{R}$ & $\mathrm{F}$ & $\mathrm{FC}$ & $B$ & OM \\
\hline 43. Rufous Treepie & Dendrocitta vagabunda & $\mathrm{R}$ & Lf & $\mathrm{FC}$ & $\mathrm{B}$ & $\mathrm{OM}$ \\
\hline 44. White-bellied Drongo & Dicrurus caerulescens & $\mathrm{R}$ & Lf & $\mathrm{FC}$ & NB & IN \\
\hline 45. Black Drongo & Dicrurus macrocercus & $\mathrm{R}$ & $\mathrm{F}$ & $\mathrm{FC}$ & $\mathrm{B}$ & IN \\
\hline 46. Eurasian Golden Oriole & Oriolus oriolus & LM & Lf & 0 & NB & $\mathrm{FR}(?)^{*}$ \\
\hline 47. Small Minivet & Pericrocotus cinnamomeus & $\mathrm{R}$ & $\mathrm{F}$ & $\mathrm{FC}$ & $\mathrm{B}$ & IN \\
\hline 48. White-throated Fantail & Rhipidura albicollis & $\mathrm{R}$ & Lf & $\mathrm{FC}$ & NB & IN \\
\hline 49. White-browed Fantail & Rhipidura aureola & $\mathrm{R}$ & Lf & $\mathrm{FC}$ & NB & IN \\
\hline 50. Asian Paradise-flycatcher & Terpsiphone paradisi & V & $\mathrm{Sc}$ & $r$ & NB & IN \\
\hline \multicolumn{7}{|l|}{ Muscicapidae } \\
\hline 51. Brown Rockchat & Cercomela fusca & $\mathrm{R}$ & $\mathrm{F}$ & $\mathrm{FC}$ & NB & IN \\
\hline 52. Oriental Magpie Robin & Copsychus saularis & $\mathrm{R}$ & Lf & $\mathrm{FC}$ & NB & IN \\
\hline 53. Isabelline Wheatear & Oenanthe isabellina & WM & $\mathrm{Sc}$ & $\mathrm{O}$ & NB & IN \\
\hline 54. Black Redstart & Phoenicurus ochruros & WM & Lf & 0 & NB & IN \\
\hline 55. Common Redstart & Phoenicurus phoenicurus & WM & $\mathrm{Sc}$ & $\mathrm{O}$ & NB & IN \\
\hline 56. Jerdon's Bushchat & Saxicola jerdoni & $\mathrm{R}$ & $\mathrm{Sc}$ & $\mathrm{FC}$ & NB & IN \\
\hline 57. Common Stonechat & Saxicola torquata & WM & $\mathrm{Sc}$ & 0 & NB & IN \\
\hline 58. Indian Robin & Saxicoloides fulicata & $\mathrm{R}$ & $\mathrm{F}$ & $\mathrm{FC}$ & $\mathrm{B}$ & IN \\
\hline \multicolumn{7}{|l|}{ Sturnidae } \\
\hline 59. Bank Myna & Acridotheres ginginianus & $\mathrm{R}$ & $\mathrm{F}$ & $\mathrm{FC}$ & B & $\mathrm{OM}$ \\
\hline 60. Common Myna & Acridotheres tristis & $\mathrm{R}$ & $\mathrm{F}$ & $\mathrm{FC}$ & $\mathrm{B}$ & $\mathrm{OM}$ \\
\hline 61. Brahminy Starling & Sturnus pagodarum & $\mathrm{R}$ & $\mathrm{F}$ & $\mathrm{FC}$ & $\mathrm{B}$ & $\mathrm{OM}$ \\
\hline \multicolumn{7}{|l|}{ Paridae } \\
\hline 62. Great Tit & Parus major & $\mathrm{R}$ & Lf & $\mathrm{FC}$ & $?$ & IN \\
\hline 63. White-naped Tit & Parus nuchalis & LM & $\mathrm{Sc}$ & $\mathrm{C}$ & B & IN \\
\hline \multicolumn{7}{|l|}{ Hirundinidae } \\
\hline 64. Red-rumped Swallow & Hirundo daurica & $\mathrm{R}$ & $\mathrm{La}$ & $\mathrm{FC}$ & B & IN \\
\hline 65. Wire-tailed Swallow & Hirundo smithii & $\mathrm{R}$ & Lf & $\mathrm{FC}$ & $\mathrm{B}$ & IN \\
\hline 66. Plain Martin & Riparia paludicola & $\mathrm{R}$ & Lf & FC & $?$ & IN \\
\hline \multicolumn{7}{|l|}{ Pycnonotidae } \\
\hline 67. Red-vented Bulbul & Pycnonotus cafer & $\mathrm{R}$ & A & $\mathrm{FC}$ & B & FR \\
\hline 68. White-eared Bulbul & Pycnonotus leucotis & $\mathrm{R}$ & Lf & $\mathrm{FC}$ & $\mathrm{B}$ & IN $(?)^{*}$ \\
\hline \multicolumn{7}{|l|}{ Cisticolidae } \\
\hline 69. Rufous-fronted Prinia & Prinia buchanani & $\mathrm{R}$ & Lf & $\mathrm{FC}$ & B & IN \\
\hline 70. Grey-breasted Prinia & Prinia hodgsonii & $\mathrm{R}$ & $\mathrm{F}$ & FC & $\mathrm{B}$ & IN \\
\hline 71. Plain Prinia & Prinia inornata & $\mathrm{R}$ & $\mathrm{F}$ & $\mathrm{FC}$ & $\mathrm{B}$ & IN \\
\hline 72. Ashy Prinia & Prinia socialis & $\mathrm{R}$ & $\mathrm{F}$ & $\mathrm{FC}$ & $\mathrm{B}$ & IN \\
\hline Zosteropidae & & & & & & \\
\hline 73. Oriental White-eye & Zosterops palpebrosus & $\mathrm{R}$ & Lf & $\mathrm{FC}$ & $?$ & IN \\
\hline Sylviidae & & & & & & \\
\hline 74. Paddyfield Warbler & Acrocephalus agricola & WM & Lf & $\mathrm{O}$ & NB & IN \\
\hline 75. Common Tailorbird & Orthotomus sutorius & $\mathrm{R}$ & $\mathrm{F}$ & $\mathrm{FC}$ & $B$ & IN \\
\hline 76. Lesser Whitethroat & Sylvia curruca & WM & Lf & $\mathrm{O}$ & NB & IN \\
\hline 77. Yellow-billed Babbler & Turdoides affinis & $\mathrm{R}$ & Lf & $\mathrm{FC}$ & NB & IN \\
\hline 78. Common Babbler & Turdoides caudatus & $\mathrm{R}$ & $\mathrm{F}$ & $\mathrm{FC}$ & $\mathrm{B}$ & IN \\
\hline 79. Large Grey Babbler & Turdoides malcolmi & $\mathrm{R}$ & $\mathrm{La}$ & $\mathrm{FC}$ & $\mathrm{B}$ & IN \\
\hline 80. Jungle Babbler & Turdoides striatus & $\mathrm{R}$ & $\mathrm{F}$ & $\mathrm{FC}$ & $\mathrm{B}$ & IN \\
\hline Alaudidae & & & & & & \\
\hline 81. Ashy-crowned Sparrow Lark & Eremopterix grisea & $\mathrm{R}$ & $\mathrm{F}$ & $\mathrm{FC}$ & B & IN $(?)^{*}$ \\
\hline 82. Indian Bushlark & Mirafra erythroptera & $\mathrm{R}$ & Lf & $\mathrm{FC}$ & $\mathrm{BP}$ & IN \\
\hline Nectariniidae & & & & & & \\
\hline 83. Purple Sunbird & Nectarinia asiatica & $\mathrm{R}$ & $\mathrm{La}$ & $\mathrm{FC}$ & B & NR \\
\hline Passeridae & & & & & & \\
\hline 84. Indian Silverbill & Lonchura malabarica & $\mathrm{R}$ & Lf & $\mathrm{FC}$ & B & GR \\
\hline 85. White Wagtail & Motacilla alba & WM & $\mathrm{Sc}$ & 0 & NB & IN \\
\hline 86. White-browed Wagtail & Motacilla maderaspatensis & $\mathrm{R}$ & Lf & $\mathrm{FC}$ & $?$ & IN \\
\hline 87. House Sparrow & Passer domesticus & $\mathrm{R}$ & $\mathrm{F}$ & $\mathrm{FC}$ & $\mathrm{B}$ & GR \\
\hline 88. Chestnut-shouldered Petronia & Petronia xanthocollis & $\mathrm{R}$ & $\mathrm{F}$ & $\mathrm{FC}$ & $\mathrm{B}$ & GR \\
\hline 89. Baya Weaver & Ploceus philippinus & $\mathrm{R}$ & $\mathrm{F}$ & $\mathrm{FC}$ & $\mathrm{B}$ & GR \\
\hline Fringillidae & & & & & & \\
\hline 90. Crested Bunting & Melophus lathami & $\mathrm{V}$ & $\mathrm{Sc}$ & $r$ & NB & GR \\
\hline
\end{tabular}

$(?)^{\star}$ - authors are not sure of feeding habit. 


\section{Common Vegetation}

The THf covers mainly tropical thorn-scrub type vegetation. It is characterized by low altitude hill vegetation. Scrubs are dominant species of this forest. One grassland occasionally present on small part of plain areas. Shrub species are mainly mixed thorny type, which is dominantly present in all parts of the forest. Xerophyte vegetation is dominant. Agro-ecosystems exist at the peripheral areas of the forest. Anogeissus latifolia, Acacia chundra, Bauhinia recemosa, Butea monosperma and Sterculia urens are major trees. Achyranthus aspera, Adhatoda vasica, Calotropis gigantea, Maytenus emarginata, Zizyphus mauritiana and Abrus precatorius, Asparagus racemosus, Cuscuta reflexa, Pedalium murex are common shrubs and climbers. Among the herbs Andrographis paniculata, Bergia capensis, Cassia auriculata, Enicostemma hyssopifolium, Datura metel are common.

\section{Methods}

The study was conducted from early December 2006 to late November 2008. Birds were observed from early morning 0600hr till noon and approximately total 576 man hours were spent. Field work was conducted weekly during the study period in four sites within the THf.

After considering all the available methods, the variable width line transect method described by Burnham et al. (1981) was adopted, in which, the observer walks along a predetermined route at $0.58 \mathrm{~km} / \mathrm{hr}$ fixed speed, counting the birds seen or heard $10 \mathrm{~m}$ on both sides of the path. Whenever a bird was sighted, it was identified up to species and details like the number of birds, and habitat type were recorded. Birds were identified using binoculars (8X40) and with the help of field guide (Grimmett et al. 1999; Ali 2002). Bird taxonomy and names follows Grimmett et al. (1998) and characteristic and specific calls of the birds described by Whistler (1940), Dharamkumarsinhji (1954), Woodcock (1980) and Ali \& Ripley (1987) were followed for locating and identifying bird species.

\section{Status}

Resident: Resident (R) - Resident throughout the year, Local Migrant (LM) - Resident with some local movement, WM - Winter Migrant, MM - Monsoon Migrant, PM - Passage Migrant, Vagrant (V) - Vagrant with only a single or a couple of records, depending on movement and seasonality of occurrence.

Abundance: Abundant $(A)$ - Mean population is more than 100, Less abundant (La) - Mean population is 50 to 100 , Frequent $(F)$ - Mean population is 25 to 50 , Less frequent (Lf) - Mean population is 5 to 25 , Scarce (Sc) - Mean population is less than 5.

Occurrence: Out of 24 visits: Fairly Common (FC) - Sighted on 22 to 24 visits, Common (C) - Sighted on 14 to 21 visits, Occasional (O) - Sighted on 7 to 13 visits, Rare (r) - Sighted on less than 7 visits.

Breeding: Breeder (B) - Birds observed to breed or showing evidence (nests or newly fledged chick(s) recorded) of breeding in THf. Non-breeder (NB), Breeding Probable (BP) - Birds that were observed to breed throughout Gujarat yet no evidence of their breeding observed in THf, Breeding Possible (PB) and (?) - Not confirmed.

Feeding habit: Based on observation: Insectivore (IN), Frugivore (FR), Granivore (GR), Piscivore (PI), Omnivore (OM), Carnivore (CR).

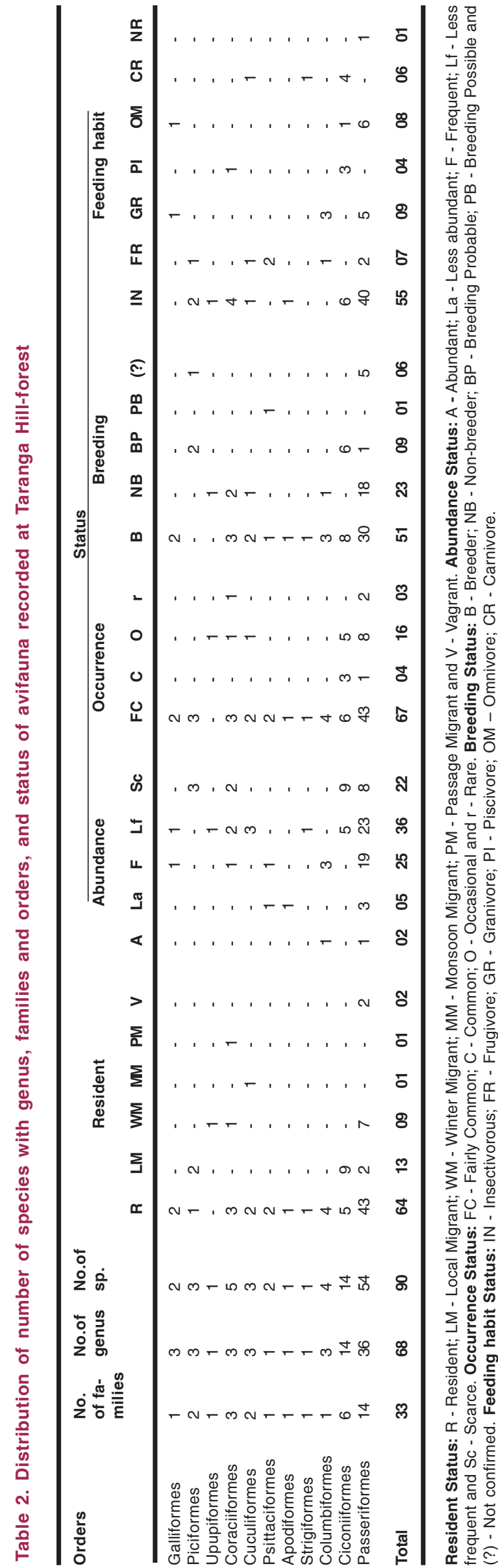




\section{Results}

Taranga Hill-forest has atleast 90 species of birds belonging to 11 orders, 33 families and 68 genera (Table 1). Common Hoopoe, Blue-cheeked Bee-eater, Isabelline Wheatear, Black Redstart, Common Redstart, Common Stonechat, Paddyfield Warbler, Lesser Whitethroat and White Wagtail were winter migrants; Pied-crested Cuckoo was monsoon migrant and European Roller was passage migrant, whereas Asian Paradise-flycatcher and Crested Bunting were vagrants. Red-vented Bulbul and Rock Pigeon were most abundant. Passage migrant and vagrant species were rare. According to Grimmett et al. (1998), White-naped Tit (Parus nuchalis) a globally threatened and endemic resident has been found as local migrant, scarce in number, common in occurrence and breeder in the tropical thorn-scrub forest habitat of THf. Plum-headed Parakeet may be a breeding possible species. Passeriformes was the largest family the next being Ciconiiformes. Distribution of number of species with genus, families and orders, and status of avifauna recorded at Taranga Hill-forest is presented in Table 2.

\section{Discussion}

So far, 146 bird species only have been recorded in Aravallis by different authors. In comparison to other places studied during last decades avian diversity is observed poor, because Aravallis are not on the migratory route or landing site of migratory birds (Prakash \& Singh 1995). In addition, anthropogenic factors, presence of predators and loss of vegetation may be having a telling effect. In Abu hill (Aravalli ranges) area also, Prakash \& Singh (1995) reported a similar condition. All common residents appear to be adapted to the prevailing conditions. Insectivorous birds were recorded more throughout the year.

\section{References}

Ali, S. (2002). The Book of Indian Birds. 13th Edition, Oxford University Press, New York, Ivii+326pp.

Ali, S. \& S.D. Ripley (1987). Compact Handbook of the Birds of India and Pakistan, Together with those of Bangladesh, Nepal, Bhutan and Sri Lanka. Oxford University Press, New Delhi, 737pp+104plates.
Burnham, K.P., D.R. Anderson \& J.L. Laake (1981). Line transect estimation of bird population density using the Fourier series, pp. 466-482. In: Ralph, C.J. \& M.J. Scott (eds.). Estimating the Terrestrial Birds. Studies in Avian Biology No.6. Cooper Ornithological Society.

Champion, H.G. \& S.K. Seth (1968). A Revised Survey of the Forest Types of India. Government of India Press, New Delhi.

Daniels, R.J.R. (1989). A conservation strategy for the birds of the Uttara Kannada District. Ph.D. Thesis. Indian Institute of Science, Bangalore.

Daniels, R.J.R. (1996). Landscape ecology and conservation of birds in the Western Ghats, South India. Ibis 138: 64-69.

Daniels, R.J.R. (1997). A Field Guide to the Birds of South Western India. Oxford University Press, New Delhi, 217pp.

Dharamkumarsinhji, R.S. (1954). Birds of Saurashtra, India. Dilbahar, Bhavnagar, Saurashtra, India, 561pp.

Furness, R.W. \& J.J.D. Greenwood (1993). Birds as a Monitor of Environmental Change. Chapman and Hall, London.

Gill, F.B. (1994). Ornithology. 2nd edition, New York.

Gokula, V. \& L. Vijayan (1996). Birds of Mudumalai Wildlife Sanctuary. Forktail 12: 107-117.

Grimmett, R., C. Inskipp \& T. Inskipp (1998). Birds of the Indian Subcontinent. Oxford University Press, New Delhi, 888pp.

Grimmett, R., C. Inskipp \& T. Inskipp (1999). Pocket Guide to the Birds of Indian Subcontinent. Oxford University Press, New Delhi, 384pp.

Johnsingh, A.J.T. \& J. Joshua (1994). Avifauna in three vegetation types on Mundanthurai plateau, South India. Journal of Tropical Ecology 10: 323-335.

Johnsingh, A.J.T., N.H. Martin, J. Balasingh \& V. Chelladurai (1987). Vegetation and avifauna in a thorn scrub habitat in South India. Journal of Tropical Ecology 28: 2234.

Katti, M.V. (1989). Birds communities of lower Dachigam. Valley, Kashmir. M.Sc. dissertation, Saurashtra University, Rajkot.

Prakash, I. \& P. Singh (1995). Some observation on the birds of Abu Hill, Aravalli Ranges. Pavo 27: 99-110.

Ramakrishnan, P. (1983). Environmental studies on the birds of Malabar Forest. Ph.D. Thesis, University of Calicut.

Schwartz, C.W. \& E.R. Schwartz (1951). An ecological reconnaissance of the pheasants of Hawaii. Auk 68: 281-314.

Sundaramoorthy, T. (1991). Ecology of terrestrial birds in Keoladeo National Park, Bharatput. Ph.D. Thesis, University of Bombay.

Whistler, H. (1940). The ornithological survey of Jodhpur state. Journal of Bombay Natural History Society XL: 214-235.

Woodcock, M. (1980). Collins Hand Guide to the Birds of the Indian Sub-continent. Collins St. Jame's Place, London, 176pp. 